\title{
DOZEN: Cross-Domain Zero Shot Named Entity Recognition with Knowledge Graph
}

\author{
Hoang Van Nguyen \\ PayPal \\ Singapore, Singapore \\ fgelli@paypal.com
}

\author{
Francesco Gelli \\ PayPal \\ Singapore, Singapore \\ vanguyen@paypal.com
}

\author{
Soujanya Poria \\ Singapore University of Technology \\ and Design \\ Singapore, Singapore \\ sporia@sutd.edu.sg
}

\begin{abstract}
With the new developments of natural language processing, increasing attention has been given to the task of Named Entity Recognition (NER). However, the vast majority of work focus on a small number of large-scale annotated datasets with a limited number of entities such as person, location and organization. While other datasets have been introduced with domain-specific entities, the smaller size of these largely limits the applicability of state-ofthe-art deep models. Even if there are promising new approaches for performing zero-shot learning (ZSL), they are not designed for a cross-domain settings. We propose Cross Domain Zero Shot Named Entity Recognition with Knowledge Graph (DOZEN), which learns the relations between entities across different domains from an existing ontology of external knowledge and a set of analogies linking entities and domains. Experiments performed on both large scale and domain-specific datasets indicate that DOZEN is the most suitable option to extracts unseen entities in a target dataset from a different domain.
\end{abstract}

\section{CCS CONCEPTS}

- Computing methodologies $\rightarrow$ Natural language processing; Information extraction; Knowledge representation and reasoning; Learning paradigms.

\section{KEYWORDS}

named entity recognition;zero-shot learning;natural language processing;knowledge graph;cross-domain machine learning

ACM Reference Format:

Hoang Van Nguyen, Francesco Gelli, and Soujanya Poria. 2021. DOZEN: Cross-Domain Zero Shot Named Entity Recognition with Knowledge Graph. In Proceedings of the 44th International ACM SIGIR Conference on Research and Development in Information Retrieval (SIGIR '21), July 11-15, 2021, Virtual Event, Canada. ACM, New York, NY, USA, 5 pages. https://doi.org/10.1145/ 3404835.3463113

\section{INTRODUCTION}

For Named Entity Recognition (NER), many approaches have recently been proposed to leverage the expressive power of language

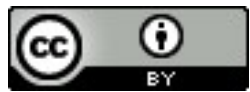

This work is licensed under a Creative Commons Attribution International 4.0 License. SIGIR '21, July 11-15, 2021, Virtual Event, Canada. (C) 2021 Copyright held by the owner/author(s). ACM ISBN 978-1-4503-8037-9/21/07.

https://doi.org/10.1145/3404835.3463113 models for extracting entities [16]. However, such solutions require large-scale annotated datasets, such as CoNLL-2003 [14] or Onto Notes 5.0 [4], which contain thousands of sentences with manually annotated entities such as person, location and organization. Such solutions generalize poorly in cross-domain scenarios, where: 1) the entities are domain-specific, such as chemical element or artist and 2) there is no large-scale annotated dataset. Recently, Liu et al. [8] released five cross-domain datasets for the domains of Politics, Natural Science, Music, Literature and Artificial Intelligence. However, even if some entities are shared across the five domains, the datasets also have a large number of domain-specific entities. Second, the cross-domain datasets are much smaller than the commonly used large-scale dataset, making the adoption of the most recent state-of-the-art supervised methods unsuitable.

To address the recognition of unseen entities, a common solution is Zero-Shot Learning (ZSL). Several works have been recently proposed to perform ZSL for NER [5, 6], mostly using some kind of metric learning to compare the sentence tokens with the entities [2]. However, the majority of these solutions assume that the training and evaluation set belong to the same domain, while in cross-domain NER, both source and target datasets differ in both the set of labels and the domain. For example, Jia et al. [6] use source-target combinations either from the same domain of biology [10], or from general-domain datasets such as CoNLL-2003 and Twitter [9], which differ only for entity Miscellaneous. The crossdomain settings has two main challenges. First, the unseen entities in the target domain are often semantically very different from the entities of the source domain. For example, the entity enzyme in the science domain is an extremely different concept from entities in the domain of music. Second, the entities that we expect to be recognized in an input sentence depend on the domain from which the sentence was sampled from. For example, given the sentence Radium was discovered by Marie Curie for which she earned her Nobel Prize in 1911., Marie Curie can be in most cases annotated as Person, but in the domain of Science, the entity Scientist will instead be the most appropriate prediction. Standard methods for ZSL in NER don't address these two issue, resulting in low performance when performing inference on a target dataset from a different domain.

To address these limitations we propose Cross-Domain Zero Shot Named Entity Recognition with Knowledge Graph (DOZEN). The model learns the relations between entities across different domains from an existing ontology of external knowledge and a set of analogies linking entities and domains. By leveraging entities which are shared from source and target domain, the ontology acts as bridge between the two domains, as the entities are connected within each others in the graph. For example, we can consider the 


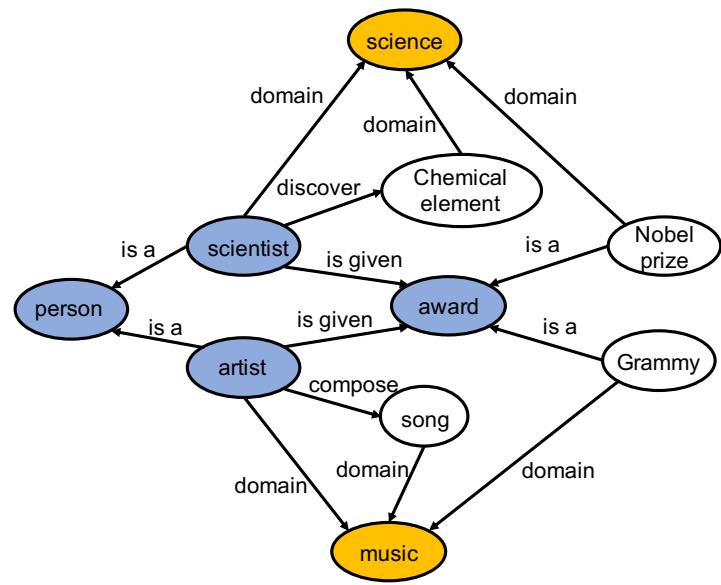

Figure 1: Example of knowledge graph: the nodes in blue and yellow are entities and domains respectively

following two sentences from the two domains of science and music respectively, and the knowledge graph in Figure 1:

(1) Radium [chemical element] was discovered by Marie Curie [scientist] for which she earned her Nobel Prize [award] in 1911.

(2) Donald Glover [artist] composed "This is America" [song] and was awarded the Grammy [award] in 2018.

From this figure we can see that the entity Person can link the two entities scientist and artist from the two different domains of science and music. A similar behaviour can be observed for shared entity award and the domain-specific entities Nobel Prize and Grammy. Thanks to these observations, even if DOZEN was never trained on the music domain, it can still recognize the entity Grammy. To learn rich graph embedding that capture these relations, DOZEN employs a Recurrent Graph Convolutional Neural Networks (RGCN) and regularizes the embedding with a novel auxiliary tasks of analogy classification, which captures the analogies of the entities across domains. In the example of Figure 1, such analogies are science : scientist :: music : artist and science : Nobel prize :: music : Grammy.

To the best of our knowledge, this is the first solution to address the task of cross-domain NER by performing ZSL on a knowledge graph. Experiments performed on public datasets illustrate that our solution not only better extracts unseen entities, but also have better performances on the seen entities in sentences from the target domain.

\section{DOZEN-METHODOLOGY}

In this section we describe the various components of DOZEN. The first input of the model is a sentence, represented as a sequence of tokens. We assume that each token has an embedding representation, which we indicate as $\mathbf{x}_{\mathbf{i}}, i \in 1, \ldots, K$, with $K$ equals to the number of tokens in the input sentence. The second input is the source and target domain, which we use to sample the relevant knowledge graph. We use $N$ to indicate the total number of entities, including both source and target domain.

The architecture of DOZEN is designed to learn rich token and entity representation as well as integrate the domain information in the token embedding. The three main components of the model are: 1) a sentence network that learns domain-independent token features, 2) a graph network to learn entity representation for source and target domain and 3) a domain integration network that integrates the domain information into the token representation, generating domain-specific token features. We provide more details on each component in the rest of this section. The overall architecture is illustrated in Figure 2.

\subsection{Domain Independent Token Features: Sentence Network}

Inspired by the most recent works on NER, we adopt a network based on BERT [1] to obtain a contextualized representation of words and entities [16]. For this component, which we indicate as $\phi$, we take inspiration from the LUKE architecture [16], since it is the current state-of-the-art of traditional NER. LUKE outputs contextualized representation for each word and entity. The LUKE model is trained on a set of downstream tasks, such as NER, Q\&A, relation classification and entity typing. Since the LUKE network has no knowledge of what is the domain of the input sentence, we use the output representation as domain independent token features. We denote such vector as $\mathbf{h}_{\mathbf{i}}=\phi\left(\mathbf{x}_{\mathbf{i}}\right)$, where $i \in 1, \ldots ., K$. To facilitate learning, we use the pre-trained network provided by [16].

\subsection{Entity Features: Graph Network}

In order to take advantage of the knowledge incorporated in the ontology, we adopt a graph network to learn entity features that capture the proximity and relations between the graph nodes. The first step consists in sampling a subset ontology given the source and target domain. For this goal, we use the source and target entities as seed nodes and propagate one level in the ontology graph to retrieve all the neighbor nodes. We denote the sampled graph as $\mathcal{G}$, and use $\mathbf{g}_{\mathbf{i}}, i \in 1, \ldots, N$ to indicate the vector representation of the $N$ entities. Given the recent advances in graph learning, we use a recurrent graph neural network (RGCN) [12] to learn the entity representations: $\mathbf{e}_{\mathbf{i}}=\psi\left(\mathbf{g}_{\mathbf{i}}\right)$, where $i \in 1, \ldots, N$. We introduce a special entity vector $\mathbf{e}_{\emptyset}$ for the outside-entity tokens, which don't have any entity. Since we use RGCN, this node representation captures the patterns observable in the local neighborhoods, such as the relation between musical artist and scientist in Figure 1. Same as in [3], we pre-train the graph network on the task of link prediction for better performance.

\subsection{Domain Integration Network}

Since the input to the sentence network $\phi$ is only the input sentence, the output representation is domain independent. We compute the domain-specific features with a multi-head attention followed by a concatenation operator:

$$
\mathbf{d}_{\mathbf{i}}=\gamma\left(\mathbf{h}_{\mathbf{i}}\right)=M H A\left(\mathbf{h}_{\mathbf{i}}, \mathbf{e}_{\mathbf{1}}, \mathbf{e}_{2}, \ldots, \mathbf{e}_{\mathbf{N}}, \mathbf{e}_{\emptyset}\right) \oplus \mathbf{h}_{\mathbf{i}} \oplus \mathbf{g}^{*}
$$

where $M H A\left(\mathbf{h}_{\mathbf{i}}, \mathbf{e}_{1}, \mathbf{e}_{2}, \ldots, \mathbf{e}_{\mathbf{N}}, \mathbf{e}_{\emptyset}\right)$ is the result of multi-head attention [15]. $\mathbf{h}_{\mathbf{i}}$ acts as the attention query and the label entity vectors $\mathbf{e}_{1}, \mathbf{e}_{2}, \ldots, \mathbf{e}_{N}, \mathbf{e}_{\emptyset}$ acts as the attention keys and values where $\oplus$ is the concatenation operator. The intuition is that most compatible 


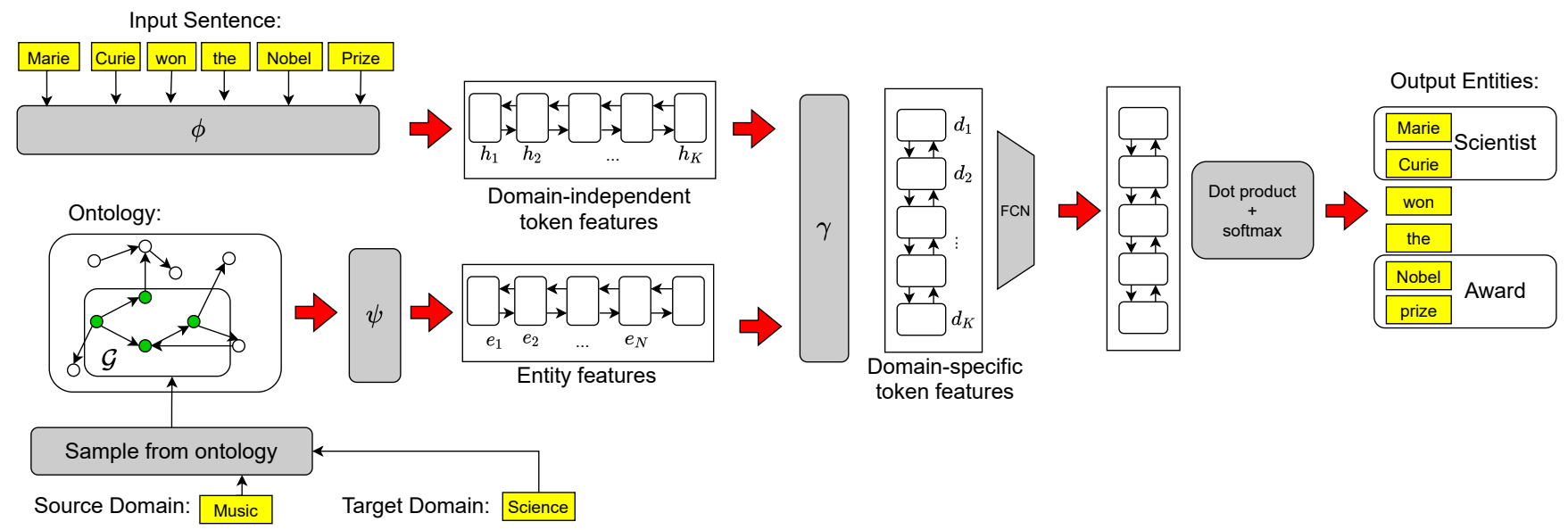

Figure 2: Architecture of DOZEN. We use yellow for the inputs and output, while we use white for the intermediate features in white and grey for the network components

label entity will generate highest attention values and its vector will contributes the highest weight in the domain-specific features.

In order to maintain the domain-independent information, $\mathbf{h}_{\mathbf{i}}$ is concatenated to the result of multi-head attention, together with $g^{*}$, which is computed as:

$$
g^{*}= \begin{cases}g_{j} & \text { if } i \text {-th token } \in \mathcal{G} \\ z e r o s & \text { if } \text { otherwise }\end{cases}
$$

In other words, if the $i$-th token corresponds to a node in in the domain-specific graph $\mathcal{G}$, then we concatenate the corresponding entity vector. With this approach we aim at facilitating the prediction of the correct entity by enriching the feature $\mathbf{d}_{\mathbf{i}}$ with relevant information.

A fully connected layer FCN reduces the dimensionality of $\mathbf{d}_{\mathbf{i}}$ to a more compact representation, before we finally compute the dot product of each token feature $\mathbf{d}_{\mathbf{i}}$ with each of the entity features $\mathbf{e}_{\mathbf{j}}$. To annotate the $i$-th token with the final prediction, we then use:

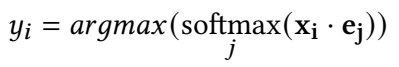

\section{DOZEN-OPTIMIZATION}

We optimize the parameters of DOZEN with a combination of multiple loss functions. We denote our source training dataset as $\mathcal{D}=\left\{\left(\mathbf{x}^{\mathbf{j}}, \hat{\mathbf{y}}^{\mathbf{j}}\right)\right\}$, where $\mathbf{x}^{\mathbf{j}}=\left[\mathbf{x}_{1}, \mathbf{x}_{2}, \ldots, \mathbf{x}_{\mathbf{K}}\right] \cdot \hat{\mathbf{y}}^{\mathbf{j}}=\left[\hat{\mathbf{y}}_{1}, \hat{\mathbf{y}}_{2}, \ldots, \hat{\mathbf{y}}_{\mathbf{K}}\right]$ is used to indicate the ground truth annotations. Following the standard approach for NER optimization, we employ the following cross entropy loss to minimize the error between the predicted tokens $\mathrm{y}^{\mathrm{j}}$ and the labels $\hat{\mathbf{y}}^{\mathrm{j}}$ :

$$
\mathcal{L}_{\text {main }}=-\frac{1}{|\mathcal{D}|} \sum_{j, i}\left(\hat{\mathbf{y}}_{i}^{\mathbf{j}} \log \mathbf{y}_{i}^{\mathbf{j}_{i}}+\left(1-\hat{\mathbf{y}}_{i}^{\mathbf{j}}\right) \log \left(1-\mathbf{y}_{i}{ }_{i}\right)\right)
$$

\subsection{Analogy Classification}

We designed the second loss terms from the intuition that entities of multiple domains are connected by analogies. For example, entity scientist in domain science correspond to entity artist in domain music. Analogy prediction has been formalized in the NLP literature as the task of predicting what entity best suits an analogy of shape a:b::c:d [7]. Our goal is to enforce that such analogies are reflected in the graph embedding that we learn during training. In order to enforce this behavior, we introduce a new loss term as follows.

Given a tuple $\left(d_{s}, d_{t}, e_{s}, e_{x}\right)$ with the source domain $d_{s}$, a target domain $d_{t}$, two entities $e_{s}$ and $e_{x}$, with $e_{s} \in d_{s}$, the task consists in predicting if $e_{x} \in d_{t}$. For example, given (music, science, artist, scientist), we expect the model to predict a positive outcome. For this task we use an auxiliary dataset $\mathcal{D}_{\text {anlg }}=\left\{\left(d_{s}, d_{t}, e_{s}, e_{x}\right.\right.$, out $\left.)\right\}$, where out is the binary outcome.

Figure 3 illustrates the main intuition behind this task. We first retrieve the corresponding ontology vector for each entity or domain, as described in section 2.2. For tuple $\left(d_{s}, d_{t}, e_{s}, e_{x}, o u t\right)$, we obtain vectors $\left(\mathbf{d}_{\mathrm{s}}, \mathbf{d}_{\mathrm{t}}, \mathbf{e}_{\mathbf{s}}, \mathbf{e}_{\mathbf{x}}\right.$, out $)$. We then compute the vector difference $d_{s}-e_{s}$ and sum it to $d_{t}$, obtaining the expected analogy vector $\mathrm{e}^{*}$. The loss objective is computed as:

$$
\mathcal{L}_{\text {anlg }}=-\left(\text { out } * \log \left(\mathbf{e}^{*} \cdot \mathbf{e}_{\mathbf{x}}\right)+(1-\text { out }) \log \left(1-\left(\mathbf{e}^{*} \cdot \mathbf{e}_{\mathbf{x}}\right)\right)\right.
$$

By minimising this term, we encourage the graph vectors for entities with positive outcome to follow the geometric rules of analogies, while at the same time the entities with negative outcomes will assume a more distant position.

The final expression of the loss is

$$
\mathcal{L}=\mathcal{L}_{\text {main }}+\alpha \mathcal{L}_{\text {anlg }}
$$

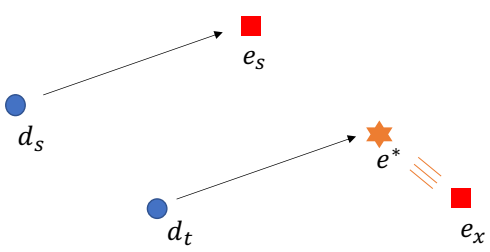

Figure 3: Geometric interpretation of the analogy classification: DOZEN either minimizes or maximises the distance between $\mathrm{e}^{*}$ and $\mathrm{e}_{\mathrm{x}}$, according to the out variable 


\section{EXPERIMENTS}

\subsection{Experiment Settings}

For domain specific datasets we use the datasets by Liu et al. [8]. We choose the three domains of science, literature and music, since they have the largest number of entities. Each domain has a training, a development and a testing set. We re-arrange the original trainvalidation-test sets to increase the amount of training data. Among these three we perform multiple experiments, where one domain is fixed as source, and a second as target, for a total of six combinations. For the source dataset we use the annotation as labels $\hat{\mathbf{y}}^{j}$, while for the target domain we assume there is no label available.

For the common-sense knowledge graph, we chose ConceptNet5.5 [13] as in [3]. Since the network contains more than 34 million edges contains, we sample a smaller ontology with a prefixed universe of the five domains in the dataset by [8]. We perform the sampling by extracting nouns and pronouns with a part-ofspeech algorithm from all sentences in the dataset, then search for nodes in ConceptNet5.5 and selecting the adjacent nodes.

We compare DOZEN with two baselines. The first is based on the state-of-the-art NER model LUKE [16]. To extend it to ZSL, we learn feature for entities, then compute the dot product with these with the token hidden states after a fully connected layers. The second baseline is an ablated version of DOZEN without analogy loss, which we name as DOZEN* ${ }^{*}$. Source code is available at ${ }^{1}$.

We used the development set for tuning the parameters and for the stopping criteria.

\subsection{Results}

Table 1 shows our experiment results in terms of macro F1 score. Results in the table are organized for three different settings, where we consider unseen and seen target entities separately, and all the target entities together.

Unseen entities (ZSL): we observe that DOZEN improves from LUKE+ZSL in 5 out of 6 domain pairs on unseen data. The improvement margin in terms of $F 1$ score is 0.04 on average, ranging from 0.148 in science-to-literature domain pair to -0.001 in music-to-literature domain pair. This confirms the importance of commonsense knowledge in ZSL.

Seen entities (Cross-domain): we can see that our model can achieve a higher F1 score across 5 in 6 domain pairs on unseen data, with average improvement margin of 0.036 . This shows that the knowledge graph and analogies do not benefit the ZSL capability only, but also help with recognizing the same source entities in sentences from the target domain.

All entities: on all entities, DOZEN improves LUKE across 5 out of 6 domain pairs with average improvement margin of 0.039 , indicating that DOZEN is the most suitable option for cross-domain NER. From the table we can see that in the science-to-music, all of the models fail to achieve ZSL, with F1 score close to 0 . In order to explain this behaviour, we followed the approach by [11], where they used the KL divergence as a measure of how the target domain differ from the source domain in terms of the distribution of concepts. We found out that among all the six pairs of domain, the

\begin{tabular}{|l|l|c|c|c|}
\hline & & All Entities & Seen Entities & Unseen Entities \\
\hline \hline \multirow{2}{*}{$\begin{array}{l}\text { S:Science } \\
\text { T:Literature }\end{array}$} & DOZEN & $\mathbf{0 . 3 7 2}$ & $\mathbf{0 . 5 6 9}$ & $\mathbf{0 . 0 9 7}$ \\
\cline { 2 - 5 } & DOZEN $^{*}$ & 0.338 & 0.563 & 0.023 \\
\cline { 2 - 5 } & LUKE+ZSL & 0.344 & 0.545 & 0.063 \\
\hline \hline \multirow{2}{*}{$\begin{array}{l}\text { S:Literature } \\
\text { T:Science }\end{array}$} & DOZEN & $\mathbf{0 . 3 2 7}$ & $\mathbf{0 . 5 3 6}$ & 0.180 \\
\cline { 2 - 5 } & DOZEN $^{*}$ & 0.316 & 0.502 & $\mathbf{0 . 1 8 7}$ \\
\cline { 2 - 5 } & LUKE+ZSL & 0.268 & 0.408 & 0.169 \\
\hline \hline \multirow{3}{*}{$\begin{array}{l}\text { S:Music } \\
\text { T:Literature }\end{array}$} & DOZEN & 0.485 & 0.616 & 0.301 \\
\cline { 2 - 5 } & DOZEN & 0.445 & 0.567 & 0.274 \\
\cline { 2 - 5 } & LUKE+ZSL & $\mathbf{0 . 4 9 5}$ & $\mathbf{0 . 6 3 3}$ & $\mathbf{0 . 3 0 2}$ \\
\hline \hline \multirow{2}{*}{$\begin{array}{l}\text { S:Literature } \\
\text { T:Music }\end{array}$} & DOZEN & $\mathbf{0 . 4 1 5}$ & $\mathbf{0 . 5 9 2}$ & $\mathbf{0 . 2 6 4}$ \\
\cline { 2 - 5 } & DOZEN & 0.355 & 0.576 & 0.098 \\
\cline { 2 - 5 } & LUKE+ZSL & 0.322 & 0.559 & 0.118 \\
\hline \hline \multirow{2}{*}{$\begin{array}{l}\text { S:Music } \\
\text { T:Science }\end{array}$} & DOZEN & $\mathbf{0 . 2 6 5}$ & $\mathbf{0 . 4 3 8}$ & $\mathbf{0 . 1 1 6}$ \\
\cline { 2 - 5 } & DOZEN & 0.253 & 0.435 & 0.104 \\
\cline { 2 - 5 } & LUKE+ZSL & 0.227 & 0.430 & 0.061 \\
\hline \hline \multirow{2}{*}{$\begin{array}{l}\text { S:Science } \\
\text { T:Music }\end{array}$} & DOZEN & 0.284 & $\mathbf{0 . 5 2 7}$ & 0.000 \\
\cline { 2 - 5 } & DOZEN & $\mathbf{0 . 2 8 8}$ & 0.525 & $\mathbf{0 . 0 1 0}$ \\
\cline { 2 - 5 } & LUKE+ZSL & 0.261 & 0.486 & 0.000 \\
\hline
\end{tabular}

Table 1: Performance of DOZEN in terms of macro F1 score

KL diverge is the highest when the source is science and the target is music, suggesting that this combination is the hardest for ZSL.

$D O Z E N^{*}$ : first, we notice that in 2 out of 6 cases, DOZEN* achieves a smaller F1 score than the baseline $L U K E+Z S L$, but $D O Z E N$ has the highest score. This indicate that the anomaly classification is a critical component of DOZEN. Second, we observe that the DOZEN has better results than the DOZEN* variation, with the exception of 2 domain pairs, namely literature-science and science-music, confirming that overall the model benefits from the analogy learning. A possible explanation for these two combinations is that since we engineered the analogies dataset ourselves, these may be biased and fail to reflect the correct analogical relationships between labels of certain domain pairs. Future works shall seek to mitigate this engineering bias and propose more robust analogies.

\section{CONCLUSION}

Most of works that do zero shot learning are not designed for crossdomain settings, where the entities domain-specific and there is no large-scale annotated dataset. Our solution, which we name Cross Domain Zero Shot Named Entity Recognition with Knowledge Graph (DOZEN), bridges the gap between the multiple domains by leveraging an external knowledge and learning to correctly classify associations. Our experiments performed on six combination of three domain-specific datasets indicate that our solution not only better extracts unseen entities, but also have better performances on the seen entities in sentences from the target domain. In the future, we plan to reduce the engineering bias from the analogy dataset and to integrate domain adaptation in the model, to address the differences in the data and label distribution across multiple domains.

\footnotetext{
${ }^{1}$ https://github.com/GelliFrancesco/DOZEN
} 


\section{REFERENCES}

[1] Jacob Devlin, Ming-Wei Chang, Kenton Lee, and Kristina Toutanova. 2019. BERT: Pre-training of Deep Bidirectional Transformers for Language Understanding. In Proceedings of the 2019 Conference of the North American Chapter of the Association for Computational Linguistics: Human Language Technologies, Volume 1 (Long and Short Papers). Association for Computational Linguistics, Minneapolis, Minnesota, 4171-4186. https://doi.org/10.18653/v1/N19-1423

[2] Alexander Fritzler, Varvara Logacheva, and Maksim Kretov. 2019. Few-Shot Classification in Named Entity Recognition Task. In Proceedings of the 34th ACM/SIGAPP Symposium on Applied Computing (Limassol, Cyprus) (SAC '19). Association for Computing Machinery, New York, NY, USA, 993-1000. https: //doi.org/10.1145/3297280.3297378

[3] Deepanway Ghosal, Devamanyu Hazarika, Abhinaba Roy, Navonil Majumder, Rada Mihalcea, and Soujanya Poria. 2020. KinGDOM: Knowledge-Guided DOMain Adaptation for Sentiment Analysis. In Proceedings of the 58th Annual Meeting of the Association for Computational Linguistics. Association for Computational Linguistics, Online, 3198-3210. https://doi.org/10.18653/v1/2020.aclmain.292

[4] Eduard Hovy, Mitchell Marcus, Martha Palmer, Lance Ramshaw, and Ralph Weischedel. 2006. OntoNotes: The 90\% Solution. In Proceedings of the Human Language Technology Conference of the NAACL, Companion Volume: Short Papers. Association for Computational Linguistics, New York City, USA, 57-60.

[5] Chen Jia, Xiaobo Liang, and Yue Zhang. 2020. Cross-domain NER using crossdomain language modeling. ACL 2019 - 57th Annual Meeting of the Association for Computational Linguistics, Proceedings of the Conference (2020), 2464-2474. https://doi.org/10.18653/v1/p19-1236

[6] Chen Jia and Yue Zhang. 2020. Multi-Cell Compositional LSTM for NER Domain Adaptation. In Proceedings of the 58th Annual Meeting of the Association for Computational Linguistics. Association for Computational Linguistics, Online, 5906-5917. https://doi.org/10.18653/v1/2020.acl-main.524

[7] Suryani Lim, Henri Prade, and Gilles Richard. 2021. Classifying and complet ing word analogies by machine learning. International fournal of Approximate Reasoning 132 (2021), 1-25. https://doi.org/10.1016/j.ijar.2021.02.002

[8] Zihan Liu, Yan Xu, Tiezheng Yu, Wenliang Dai, Ziwei Ji, Samuel Cahyawijaya Andrea Madotto, and Pascale Fung. 2020. CrossNER: Evaluating Cross-Domain Named Entity Recognition. Mlm (2020). arXiv:2012.04373 http://arxiv.org/abs/ 2012.04373
[9] Di Lu, Leonardo Neves, Vitor Carvalho, Ning Zhang, and Heng Ji. 2018. Visua attention model for name tagging in multimodal social media. ACL 2018 - 56th Annual Meeting of the Association for Computational Linguistics, Proceedings of the Conference (Long Papers) 1 (2018), 1990-1999. https://doi.org/10.18653/v1/p181185

[10] Claire Nédellec, Robert Bossy, Jin-Dong Kim, Jung-jae Kim, Tomoko Ohta, Sampo Pyysalo, and Pierre Zweigenbaum. 2013. Overview of BioNLP Shared Task 2013. In Proceedings of the BioNLP Shared Task 2013 Workshop. Association for Computational Linguistics, Sofia, Bulgaria, 1-7.

[11] Barbara Plank and Gertjan van Noord. 2011. Effective Measures of Domain Similarity for Parsing. In Proceedings of the 49th Annual Meeting of the Association for Computational Linguistics: Human Language Technologies. Association for Computational Linguistics, Portland, Oregon, USA, 1566-1576. https://www. aclweb.org/anthology/P11-1157

[12] Michael Schlichtkrull, Thomas N. Kipf, Peter Bloem, Rianne van den Berg, Ivan Titov, and Max Welling. 2018. Modeling Relational Data with Graph Convolutional Networks. In The Semantic Web, Aldo Gangemi, Roberto Navigli, MariaEsther Vidal, Pascal Hitzler, Raphaël Troncy, Laura Hollink, Anna Tordai, and Mehwish Alam (Eds.). Springer International Publishing, Cham, 593-607.

[13] Robyn Speer, Joshua Chin, and Catherine Havasi. 2017. ConceptNet 5.5: An Open Multilingual Graph of General Knowledge. In Proceedings of the Thirty-First AAAI Conference on Artificial Intelligence (AAAI'17). AAAI Press, 4444-4451.

[14] Erik F. Tjong Kim Sang and Fien De Meulder. 2003. Introduction to the CoNLL2003 Shared Task: Language-Independent Named Entity Recognition. In Proceedings of the Seventh Conference on Natural Language Learning at HLT-NAACL 2003. 142-147.

[15] Ashish Vaswani, Noam Shazeer, Niki Parmar, Jakob Uszkoreit, Llion Jones, Aidan N Gomez, Ł ukasz Kaiser, and Illia Polosukhin. 2017. Attention is All you Need. In Advances in Neural Information Processing Systems, I. Guyon, U. V. Luxburg, S. Bengio, H. Wallach, R. Fergus, S. Vishwanathan, and R. Garnett (Eds.), Vol. 30. Curran Associates, Inc. https://proceedings.neurips.cc/paper/2017/file/ 3f5ee243547dee91fbd053c1c4a845aa-Paper.pdf

[16] Ikuya Yamada, Akari Asai, Hiroyuki Shindo, Hideaki Takeda, and Yuji Matsumoto. 2020. LUKE: Deep Contextualized Entity Representations with Entity-aware Selfattention. In Proceedings of the 2020 Conference on Empirical Methods in Natural Language Processing (EMNLP). Association for Computational Linguistics, Online, 6442-6454. https://doi.org/10.18653/v1/2020.emnlp-main.523 